\title{
Diffraction of Sound Impulses on Isotropic Bodies of Spherical Form (Strict Solution)
}

\author{
A. A. Kleshchev ${ }^{*}$ A. S. Klimenkov \\ Saint Petersburg State Navy Technical University, Russia, 190008, Saint - Petersburg, Lotsmanskaya st., 3 \\ *Corresponding Author: alexalex-2@yandex.ru
}

Copyright (C) 2013 Horizon Research Publishing All rights reserved.

\begin{abstract}
In the scientific paper are submitted the calculations of form and duration of sound impulses with the harmonic infill, scattered from elastic spherical shell and elastic isotropic sphere, made with the use of dynamic theory of elasticity.
\end{abstract}

Keywords Sound Diffraction, Elastic Spherical Shell, Boundary Conditions, Impulse Signal, Harmonic Infill

\section{Introduction}

Considerable quantity of scientific works is devoted to sound scattering on bodies of spherical form [1 - 4]. The problem of diffraction of sound impulses with the harmonic infill on elastic spherical shell and elastic isotropic sphere is being reviewed in this paper. At the basis of the dynamic theory of elasticity are found form and duration of sound impulses scattered from elastic spherical shell and elastic isotropic sphere (which in general case can be with random radius, but in this paper both of sphere and shell (outer radius) got $\left.R_{2}=4,0 \mathrm{~m}\right)$. The scattering of frequency-modulated signal (impulse) can also be found in a similar way, using the basis of the dynamic theory of elasticity, but this paper contains computed results only for signal (impulse) with the harmonic infill.

\section{The First Part of the Article Investigates the Diffraction Problem of the Impulse with the Harmonic Infill on Elastic Spherical Shell}

Let's assume that monochrome sound wave falls on elastic spherical shell (Fig. 1). To find form and duration of reflected impulses it is necessary to know angular characteristics of the scattering, which can be wrote like [5]:

$$
F(\theta)=\frac{i}{k} \sum_{m=0}^{\infty} i^{-m} \cdot a_{m} \cdot P_{m}(\operatorname{Cos}(\theta)),
$$

where: $a_{m}$ - unknown expansion coefficients, $P_{m}(\operatorname{Cos}(\theta))$ - Legendre polynomial, $k$-wave number.

In contrast to well-known solutions $[6,7]$, which are based on theory of the thin shells, in our case we will use strict approach, which based on dynamic theory of elasticity $[5,8]$, to find the numerical values of angular characteristics of the scattering.

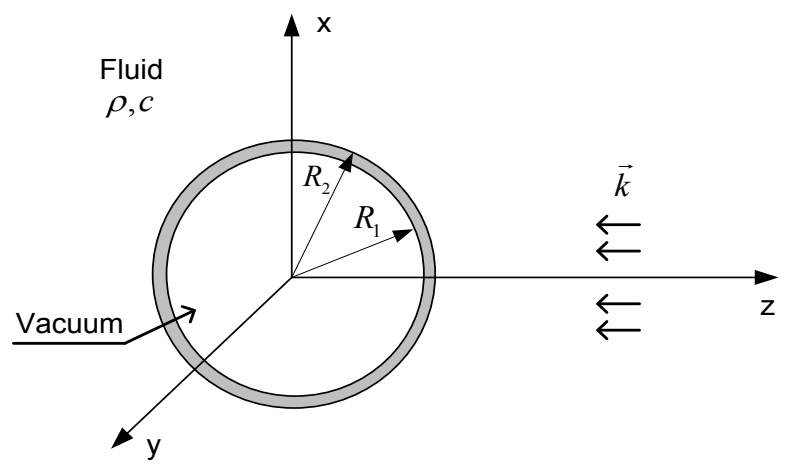

Figure 1. Elastic spherical shell.

Threshold value of $m$ take as $m=10$. Unknown expansion coefficients are determined from physical boundary conditions preset at two surfaces of the shell, which are consists in:

a) continuity of normal displacements on the border fluid and elastic spherical shell (outer radius);

b) equality of normal stress in spherical shell (on its outer border) to diffracted pressure $p_{\Sigma}$ in flow media;

c) absence of tangent stresses on inner and outer borders of the spherical shell.

Mathematically, boundary conditions look like (they acquire such form after deduction from the Lame equation with using Helmholtz theorem and generalized Hooke's law, which is invariant to the coordinate system): 


$$
\begin{gathered}
-\frac{\partial}{\partial r}\left(\Phi_{0}+\Phi_{1}\right)=-\frac{\partial \Phi_{2}}{\partial r}+\frac{1}{r} \cdot \operatorname{Ctg}(\theta) \cdot \Psi_{2}+\left.\frac{1}{r} \cdot \frac{\partial \Psi_{2}}{\partial \theta}\right|_{r=R_{2}} ; \\
\Lambda_{0} \cdot k^{2} \cdot\left(\Phi_{0}+\Phi_{1}\right)=\Lambda_{1} \cdot k_{l}^{2} \cdot \Phi_{2}+ \\
+\left.2 \cdot \mu_{1}\left(-\frac{\partial^{2} \Phi_{2}}{\partial r^{2}}+\frac{1}{r} \cdot \operatorname{Ctg}(\theta) \cdot \frac{\partial \Psi_{2}}{\partial r}-\frac{1}{r^{2}} \cdot \operatorname{Ctg}(\theta) \cdot \Psi_{2}+\frac{1}{r} \cdot \frac{\partial^{2} \Psi_{2}}{\partial \theta \partial r}-\frac{1}{r^{2}} \cdot \frac{\partial \Psi_{2}}{\partial \theta}\right)\right|_{r=R_{2}} ; \\
0=\Lambda_{1} \cdot k_{l}^{2} \cdot \Phi_{2}+ \\
+\left.2 \cdot \mu_{1}\left(-\frac{\partial^{2} \Phi_{2}}{\partial r^{2}}+\frac{1}{r} \cdot \operatorname{Ctg}(\theta) \cdot \frac{\partial \Psi_{2}}{\partial r}-\frac{1}{r^{2}} \cdot \operatorname{Ctg}(\theta) \cdot \Psi_{2}+\frac{1}{r} \cdot \frac{\partial^{2} \Psi_{2}}{\partial \theta \partial r}-\frac{1}{r^{2}} \cdot \frac{\partial \Psi_{2}}{\partial \theta}\right)\right|_{r=R_{1}} ; \\
\frac{1}{r^{2}} \cdot \operatorname{Ctg}(\theta) \cdot \frac{\partial \Psi_{2}}{\partial \theta}+\frac{1}{r^{2}} \cdot \frac{\partial^{2} \Psi_{2}}{\partial \theta^{2}}-\frac{\partial^{2} \Psi_{2}}{\partial r^{2}}-2 \cdot \frac{1}{r^{2}} \cdot \frac{\partial \Phi_{2}}{\partial \theta}-\left.\frac{1}{r^{2} \operatorname{Sin}^{2}(\theta)} \cdot \Psi_{2}\right|_{\substack{r=R_{2} ; \\
r=R_{1}}}=0
\end{gathered}
$$

$\Phi_{0}, \Phi_{1}, \Phi_{2}, \Psi_{2}$ are:

$$
\begin{gathered}
\Phi_{0}=\sum_{m=0}^{\infty} i^{-m} \cdot(2 m+1) \cdot P_{m}(\operatorname{Cos}(\theta)) \cdot j_{m}(k r) ; \\
\Phi_{1}=\sum_{m}^{\infty} a_{m} \cdot P_{m}(\operatorname{Cos}(\theta)) \cdot h_{m}^{(1)}(k r) ; \\
\Phi_{2}=\sum_{m}^{\infty} P_{m}(\operatorname{Cos}(\theta)) \cdot\left[b_{m} \cdot j_{m}\left(k_{l} r\right)+c_{m} \cdot n_{m}\left(k_{l} r\right)\right] ; \\
\Psi_{2}=\sum_{m}^{\infty} P_{m}^{(1)}(\operatorname{Cos}(\theta)) \cdot\left[d_{m} \cdot j_{m}\left(k_{t} r\right)+g_{m} \cdot n_{m}\left(k_{t} r\right)\right] ;
\end{gathered}
$$

where $P_{m}^{(1)}(\operatorname{Cos}(\theta))$ - shifted Legendre polynomial; $j_{m}(k r)$ и $n_{m}(k r)$ - Bessel functions and Neumann functions (Bessel functions of the second kind) correspondingly; $h_{m}^{(1)}(k r)$ - Hankel functions of the first kind (Bessel functions of the third kind).

Legendre polynomials are orthogonal to each other on angle interval $\theta$ from $0^{\circ}$ to $180^{\circ}$, i.e.:

$$
\int_{1}^{-1} P_{n}(\cos \theta) \cdot P_{m}(\cos \theta) d(\cos \theta)=\left\{\begin{array}{ccc}
0 & \text { when } & m \neq n \\
\frac{2}{2 m+1} & \text { when } & m=n
\end{array}\right.
$$

By substituting expressions (6) - (9) to the boundary conditions (2) - (5) with the use of property of orthogonality of Legendre polynomials (as well as shifted Legendre polynomials and derivatives from them) we will receive for each mode $m$ its system of equations, from which finds unknown expansion coefficients $a_{m}$. Using the Cramer's rule unknown expansion coefficients will be found like:

$$
a_{m}=\frac{\Delta^{\prime}}{\Delta}
$$

where $\Delta$ - system determinant, $\Delta^{\prime}-$ determinant, in which the column of unknown coefficient replaced by the column with free terms.

As an example let's see which system of equation appears for $m=3$ :

From condition (2):

$$
\begin{aligned}
& -2 \cdot i^{-3} \cdot j_{3}^{\prime}\left(k R_{2}\right)=\frac{2}{7} \cdot a_{3} \cdot h_{3}^{(1) '}\left(k R_{2}\right)- \\
& -\frac{2}{7} \cdot\left[b_{3} \cdot j_{3}^{\prime}\left(k_{l} R_{2}\right)+c_{3} \cdot n_{3}^{\prime}\left(k_{l} R_{2}\right)\right]+\frac{24}{7 R_{2}} \cdot\left[d_{3} \cdot j_{3}\left(k_{t} R_{2}\right)+g_{3} \cdot n_{3}\left(k_{t} R_{2}\right)\right]
\end{aligned}
$$


From condition (3):

$$
\begin{aligned}
& 2 \cdot \Lambda_{0} \cdot k^{2} \cdot i^{-3} \cdot j_{3}\left(k R_{2}\right)=-\Lambda \cdot k^{2} \cdot \frac{2}{7} \cdot h_{3}^{(1)}\left(k R_{2}\right)+ \\
& +\Lambda_{1} \cdot k_{l}^{2} \cdot \frac{2}{7}\left[b_{3} \cdot j_{3}\left(k_{l} R_{2}\right)+c_{3} \cdot n_{3}\left(k_{l} R_{2}\right)\right]+2 \mu\left[-\frac{2}{7} \cdot\left[b_{3} \cdot j_{3}^{\prime \prime}\left(k_{l} R_{2}\right)+c_{3} \cdot n_{3}^{\prime \prime}\left(k_{l} R_{2}\right)\right]+\right. \\
& \left.+\frac{24}{7 R_{2}} \cdot\left[d_{3} \cdot j_{3}\left(k_{t} R_{2}\right)+g_{3} \cdot n_{3}\left(k_{t} R_{2}\right)\right]-\frac{24}{7 R_{2}^{2}} \cdot\left[d_{3} \cdot j_{3}{ }^{\prime}\left(k_{t} R_{2}\right)+g_{3}^{\prime} \cdot n_{3}\left(k_{t} R_{2}\right)\right]\right]
\end{aligned}
$$

From condition (4):

$$
\begin{aligned}
& 0=\Lambda_{1} \cdot k_{l}^{2} \cdot \frac{2}{7}\left[b_{3} \cdot j_{3}\left(k_{l} R_{1}\right)+c_{3} \cdot n_{3}\left(k_{l} R_{1}\right)\right]+2 \mu\left[-\frac{2}{7} \cdot\left[b_{3} \cdot j_{3}^{\prime \prime}\left(k_{l} R_{1}\right)+c_{3} \cdot n_{3}{ }^{\prime \prime}\left(k_{l} R_{1}\right)\right]+\right. \\
& \left.+\frac{24}{7 R_{1}} \cdot\left[d_{3} \cdot j_{3}\left(k_{t} R_{1}\right)+g_{3} \cdot n_{3}\left(k_{t} R_{1}\right)\right]-\frac{24}{7 R_{1}^{2}} \cdot\left[d_{3} \cdot j_{3}^{\prime}\left(k_{t} R_{1}\right)+g_{3}^{\prime} \cdot n_{3}\left(k_{t} R_{1}\right)\right]\right] ;
\end{aligned}
$$

From condition (5);

$$
\begin{aligned}
& -\frac{288}{7 R_{2}^{2}} \cdot\left[d_{3} \cdot j_{3}\left(k_{t} R_{2}\right)+g_{3} \cdot n_{3}\left(k_{t} R_{2}\right)\right]-\frac{24}{7} \cdot\left[d_{3} \cdot j_{3}^{\prime \prime}\left(k_{t} R_{2}\right)+g_{3} \cdot n_{3}^{\prime \prime}\left(k_{t} R_{2}\right)\right]+ \\
& +\frac{48}{7 R_{2}^{2}} \cdot\left[b_{3} \cdot j_{3}\left(k_{l} R_{2}\right)+c_{3} \cdot n_{3}\left(k_{l} R_{2}\right)\right]-\frac{48}{R_{2}^{2}}\left[d_{7} \cdot j_{7}\left(k_{t} R_{2}\right)+g_{7} \cdot n_{7}\left(k_{t} R_{2}\right)\right]=0 ; \\
& -\frac{288}{7 R_{1}^{2}} \cdot\left[d_{3} \cdot j_{3}\left(k_{t} R_{1}\right)+g_{3} \cdot n_{3}\left(k_{t} R_{1}\right)\right]-\frac{24}{7} \cdot\left[d_{3} \cdot j_{3}{ }^{\prime \prime}\left(k_{t} R_{1}\right)+g_{3} \cdot n_{3}{ }^{\prime \prime}\left(k_{t} R_{1}\right)\right]+ \\
& +\frac{48}{7 R_{1}^{2}} \cdot\left[b_{3} \cdot j_{3}\left(k_{l} R_{1}\right)+c_{3} \cdot n_{3}\left(k_{l} R_{1}\right)\right]-\frac{48}{R_{1}^{2}}\left[d_{7} \cdot j_{7}\left(k_{t} R_{1}\right)+g_{7} \cdot n_{7}\left(k_{t} R_{1}\right)\right]=0 ;
\end{aligned}
$$

The touch at radial functions means the derivative by $r$.

In our case:

$$
\begin{gathered}
R_{1}=3,95 \mathrm{~m} ; R_{2}=4,0 \mathrm{~m} . \\
\Lambda_{0}=2,25 \cdot 10^{9} \mathrm{~N} / \mathrm{m}^{2} ; \Lambda_{1}=11,2 \cdot 10^{10} \mathrm{~N} / \mathrm{m}^{2} ; \mu_{1}=8,1 \cdot 10^{10} \mathrm{~N} / \mathrm{m}^{2} ;
\end{gathered}
$$

where $\Lambda_{0}$ - modulus of volume compression; $\Lambda_{1}$ - first Lame coefficient for steel; $\mu_{1}$ - rigidity modulus.

$$
\begin{gathered}
k=\frac{2 \pi \nu}{c} ; k_{l}=\frac{2 \pi \nu}{c_{l}} ; k_{t}=\frac{2 \pi \nu}{c_{t}} ; \\
c=1500 \mathrm{~m} / \mathrm{s} ; \quad c_{l}=5940 \mathrm{~m} / \mathrm{s} ; \quad c_{t}=3220 \mathrm{~m} / \mathrm{s} .
\end{gathered}
$$

Functional $F(\theta)$ is computing with angular step $h_{\Delta \theta}=5^{\circ}$ in the range $\theta=0^{\circ} \div 180^{\circ}$. Assume that falling on body impulse has harmonic infill, the falling angles are $\theta=0, \frac{\pi}{6}, \frac{\pi}{3}, \frac{\pi}{2}$. Knowing of angular characteristics of the scattering (its values) and use of the Fourier transform, allows us to find form and duration of sound impulses reflected from elastic spherical shell.
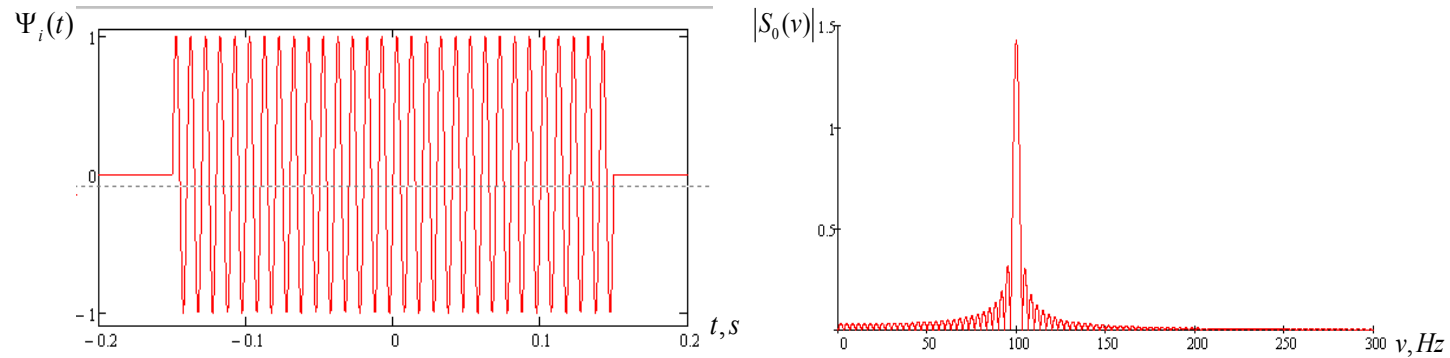

Figure 2. The falling pulse $\Psi_{i}(t)$; the spectrum module of falling pulse $\left|S_{0}(v)\right|$. 
The falling impulse with rectangular envelope and infill frequency $\omega_{0}=2 \pi v_{0}$ has the spectrum $S_{0}(2 \pi v)$ :

$$
S_{0}(2 \pi v)=\int_{-\frac{n T}{2}}^{+\frac{n T}{2}} e^{-i 2 \pi v t} \sin \left(2 \pi v_{0} t\right) d t=\frac{i v_{0}}{\pi\left(v_{0}^{2}-v^{2}\right)}(-1)^{n} \sin \left(\pi n \frac{v}{v_{0}}\right),
$$

where: $T$ - period of the harmonic signal with frequency $v_{0} ; T=1 / v_{0} ; n$ - number of periods in pulse; $v$ - current cyclic frequency.

Spectrum $S_{0}(2 \pi v)$ is bound with $\Psi_{i}(t)$ by means of reverse Fourier transform:

$$
\Psi_{i}(t)=\frac{1}{\pi} \operatorname{Re} \int_{0}^{\infty} S_{0}(2 \pi v) e^{+i 2 \pi v t} d(2 \pi v)
$$

Falling impulses with $n=30$ were used during the accounts; outer radius of the shell is $R_{2}=4.0 \mathrm{~m}$. On figures $3-6$ are represented reflected from elastic spherical shell pulses at different irradiation angles.
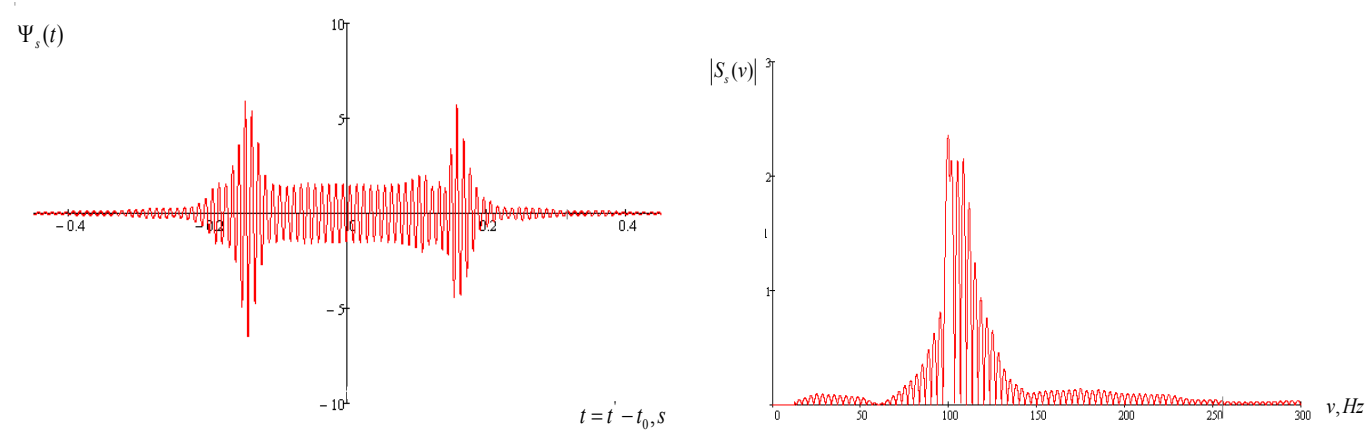

Figure 3. Impulse reflected from shell $\Psi_{s}(t)$ and its spectrum module $\left|S_{s}(v)\right|$

$$
\text { at angle } \theta=0^{\circ}
$$

$\Psi_{s}(t)$
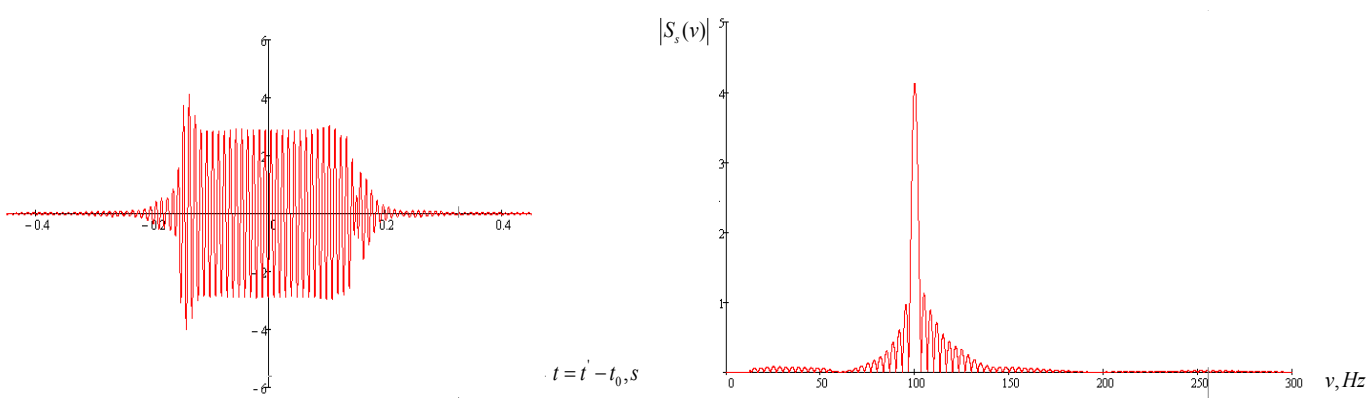

Figure 4. Impulse reflected from shell $\Psi_{s}(t)$ and its spectrum module

$\left|S_{s}(v)\right|$ at angle $\theta=30^{\circ}$

$\Psi_{s}(t)$
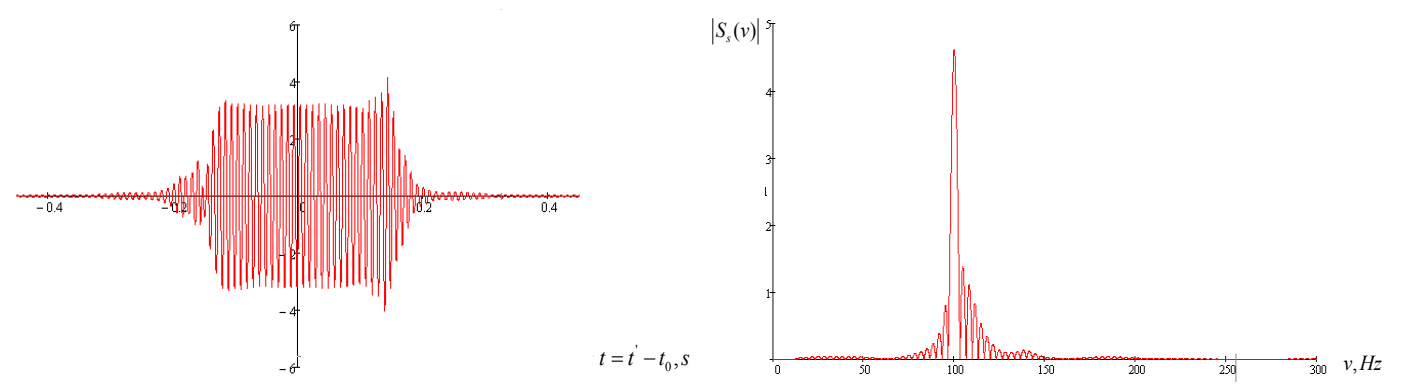

Figure 5. Impulse reflected from shell $\Psi_{s}(t)$ and its spectrum module

$$
\left|S_{s}(v)\right| \text { at angle } \theta=60^{\circ}
$$



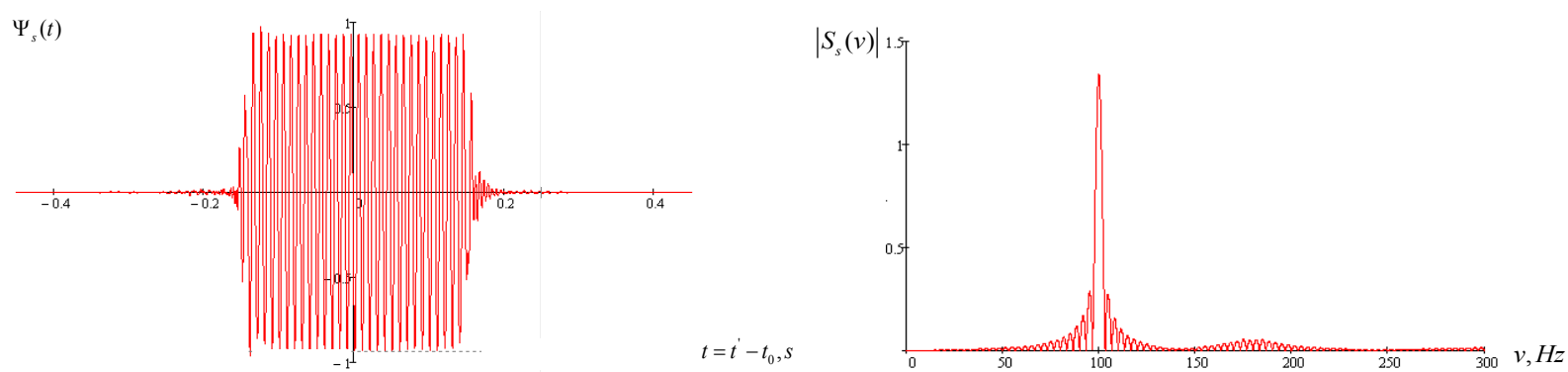

Figure 6. Impulse reflected from shell $\Psi_{s}(t)$ and its spectrum module

$$
\left|S_{s}(v)\right| \text { at angle } \theta=90^{\circ}
$$

Let's compare computed results for shell with results for sound soft ideal sphere.

$\Psi_{s}(t)$

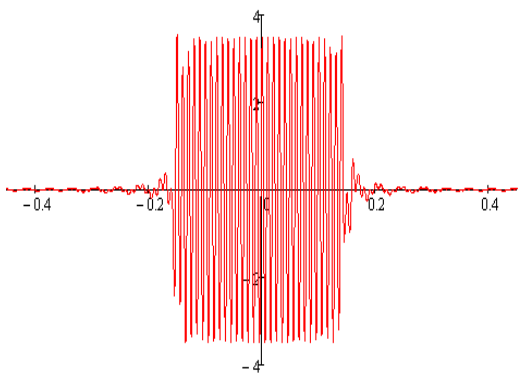

$t=t^{\prime}-t_{0}, s$
$\left|S_{s}(v)\right| r$

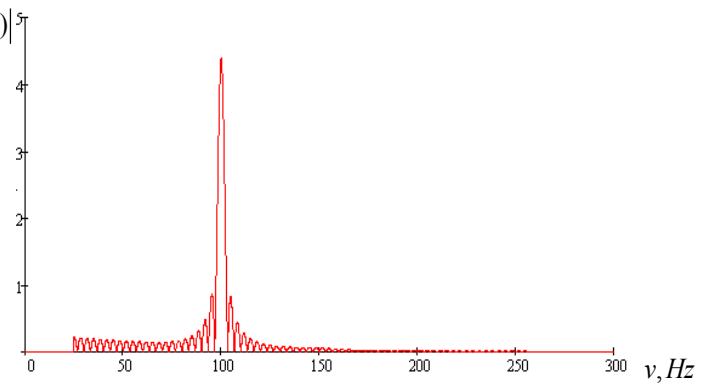

Figure 7. Impulse reflected from sound soft ideal sphere $\Psi_{s}(t)$ and its spectrum module

$$
\left|S_{s}(v)\right| \text { at angle } \theta=0^{\circ} \text {. }
$$

$\Psi_{s}(t)$

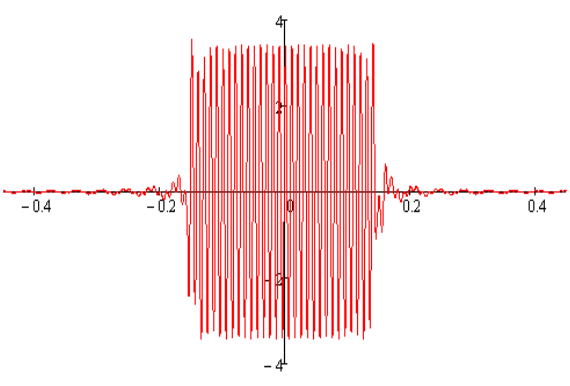

$t=t^{\prime}-t_{0}, S$

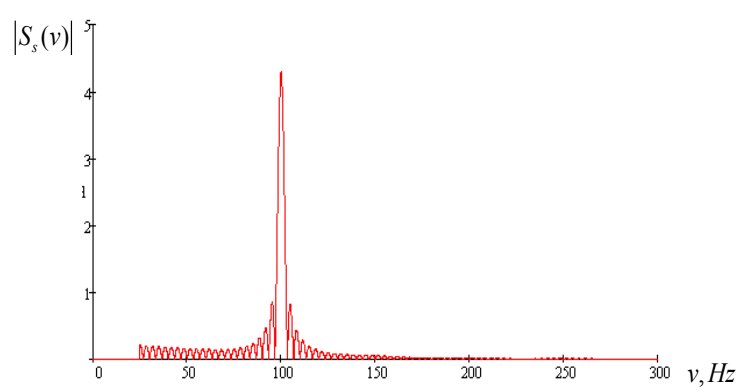

Figure 8. Impulse reflected from sound soft ideal sphere $\Psi_{s}(t)$ and its spectrum module

$$
\left|S_{s}(v)\right| \text { at angle } \theta=30^{\circ}
$$

$\Psi_{s}(t)$

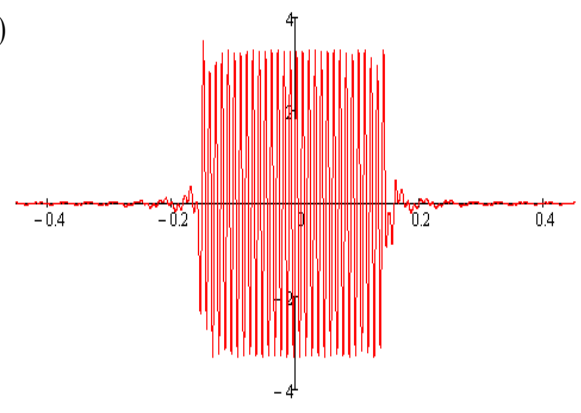

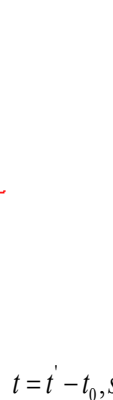

$\left|S_{s}(v)\right|$

Figure 9. Impulse reflected from sound soft ideal sphere $\Psi_{s}(t)$ and its spectrum module 


$$
\left|S_{s}(v)\right| \text { at angle } \theta=60^{\circ}
$$
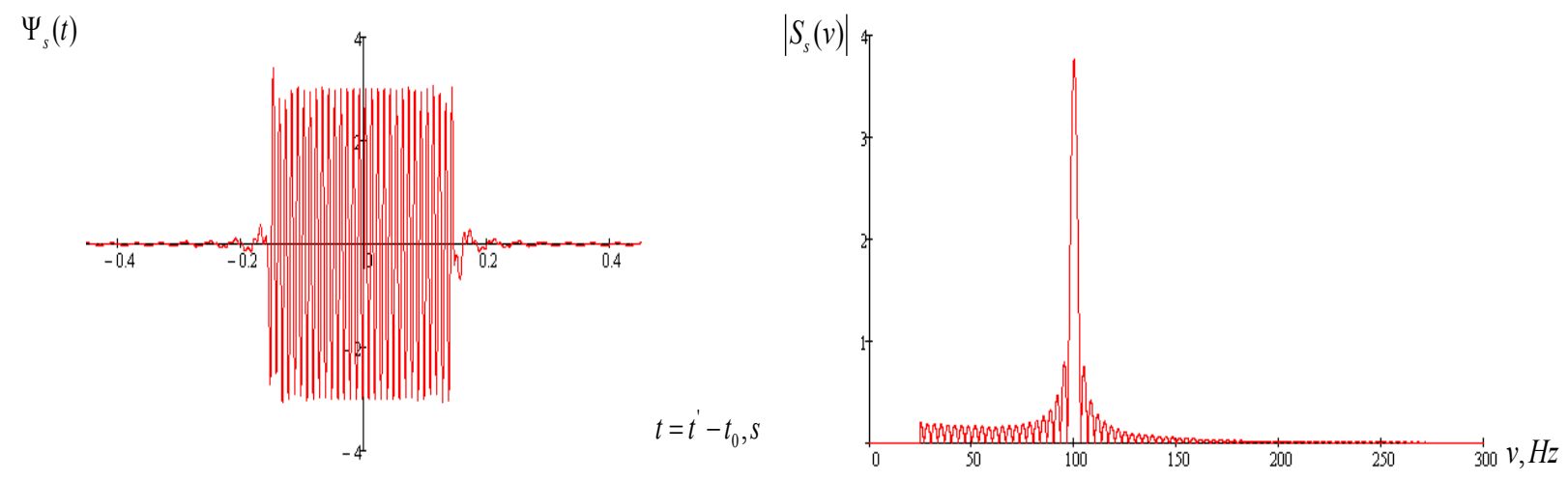

Figure 10. Impulse reflected from sound soft ideal sphere $\Psi_{s}(t)$ and its spectrum module

$$
\left|S_{s}(v)\right| \text { at angle } \theta=90^{\circ}
$$

\section{The Second Part of the Article Investigates the Diffraction Problem of the Impulse with the Harmonic Infill on Elastic Isotropic Sphere}

In case of diffraction on the elastic isotropic sphere the system of equation becomes simpler; cause of there is no more boundary condition for inner border like it was in a case of shell.

So, let's assume that monochrome sound wave falls on elastic isotropic sphere (Fig. 11). To find the numerical values of angular characteristics of the scattering for the elastic isotropic sphere, we'll also use the dynamic theory of elasticity. The radius of sphere is $R_{2}$.

To find form and duration of reflected impulses it is necessary to know angular characteristics of the scattering, which can be wrote like:

$$
F(\theta)=\frac{i}{k} \sum_{m=0}^{\infty} i^{-m} \cdot a_{m} \cdot P_{m}(\operatorname{Cos}(\theta))
$$

where $a_{m}$ - unknown expansion coefficients, $P_{m}(\operatorname{Cos}(\theta))$ - Legendre polynomial, $k$ - wave number.

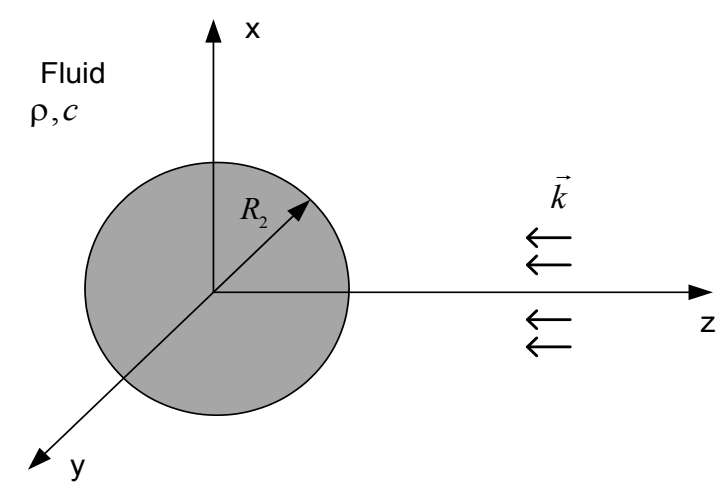

Figure 11. Elastic isotropic sphere.

Threshold value of $m$ take as $m=10$. Unknown expansion coefficients are determined from physical boundary conditions, which are consists in:

d) continuity of normal displacements on the border fluid and elastic isotropic sphere;

e) equality of normal stress on elastic isotropic sphere (outer border) to diffracted pressure $p_{\Sigma}$ in flow media;

f) absence of tangent stresses on outer border of the elastic isotropic sphere.

Mathematically, boundary conditions look like:

$$
-\frac{\partial}{\partial r}\left(\Phi_{0}+\Phi_{1}\right)=-\frac{\partial \Phi_{2}}{\partial r}+\frac{1}{r} \cdot \operatorname{Ctg}(\theta) \cdot \Psi_{2}+\left.\frac{1}{r} \cdot \frac{\partial \Psi_{2}}{\partial \theta}\right|_{r=R_{2}} ;
$$




$$
\begin{gathered}
\Lambda_{0} \cdot k^{2} \cdot\left(\Phi_{0}+\Phi_{1}\right)=\Lambda_{1} \cdot k_{l}^{2} \cdot \Phi_{2}+ \\
+\left.2 \cdot \mu_{1}\left(-\frac{\partial^{2} \Phi_{2}}{\partial r^{2}}+\frac{1}{r} \cdot \operatorname{Ctg}(\theta) \cdot \frac{\partial \Psi_{2}}{\partial r}-\frac{1}{r^{2}} \cdot \operatorname{Ctg}(\theta) \cdot \Psi_{2}+\frac{1}{r} \cdot \frac{\partial^{2} \Psi_{2}}{\partial \theta \partial r}-\frac{1}{r^{2}} \cdot \frac{\partial \Psi_{2}}{\partial \theta}\right)\right|_{r=R_{2}} \\
\frac{1}{r^{2}} \cdot \operatorname{Ctg}(\theta) \cdot \frac{\partial \Psi_{2}}{\partial \theta}+\frac{1}{r^{2}} \cdot \frac{\partial^{2} \Psi_{2}}{\partial \theta^{2}}-\frac{\partial^{2} \Psi_{2}}{\partial r^{2}}-2 \cdot \frac{1}{r^{2}} \cdot \frac{\partial \Phi_{2}}{\partial \theta}-\left.\frac{1}{r^{2} \operatorname{Sin}^{2}(\theta)} \cdot \Psi_{2}\right|_{r=R_{2}}=0
\end{gathered}
$$

$\Phi_{0}, \Phi_{1}, \Phi_{2}, \Psi_{2}$ are:

$$
\begin{gathered}
\Phi_{0}=\sum_{m=0}^{\infty} i^{-m} \cdot(2 m+1) \cdot P_{m}(\operatorname{Cos}(\theta)) \cdot j_{m}(k r) ; \\
\Phi_{1}=\sum_{m}^{\infty} a_{m} \cdot P_{m}(\operatorname{Cos}(\theta)) \cdot h_{m}^{(1)}(k r) ; \\
\Phi_{2}=\sum_{m}^{\infty} P_{m}(\operatorname{Cos}(\theta)) \cdot b_{m} \cdot j_{m}\left(k_{l} r\right) ; \\
\Psi_{2}=\sum_{m}^{\infty} P_{m}^{(1)}(\operatorname{Cos}(\theta)) \cdot d_{m} \cdot j_{m}\left(k_{t} r\right) ;
\end{gathered}
$$

where $P_{m}^{(1)}(\operatorname{Cos}(\theta))$ - shifted Legendre polynomial; $j_{m}(k r)$ - Bessel functions; $h_{m}^{(1)}(k r)$ - Hankel functions of the first kind (Bessel functions of the third kind).

By substituting expressions (18) - (21) to the boundary conditions (15) - (17) with the use of property of orthogonality of Legendre polynomials (as well as shifted Legendre polynomials and derivatives from them) we will receive for each mode $m$ its system of equations, from which finds unknown expansion coefficients $a_{m}$. As in case with shell, unknown expansion coefficients can be found by using the Cramer's rule.

As an example let's see which system of equation appears for $m=3$ :

from condition (15):

$$
-2 \cdot i^{-3} \cdot j_{3}^{\prime}\left(k R_{2}\right)=\frac{2}{7} \cdot a_{3} \cdot h_{3}^{(1)^{\prime}}\left(k R_{2}\right)-\frac{2}{7} \cdot b_{3} \cdot j_{3}^{\prime}\left(k_{l} R_{2}\right)+\frac{24}{7 R_{2}} \cdot d_{3} \cdot j_{3}\left(k_{t} R_{2}\right)
$$

from condition (16):

$$
\begin{aligned}
& 2 \cdot \Lambda_{0} \cdot k^{2} \cdot i^{-3} \cdot j_{3}\left(k R_{2}\right)=-\Lambda \cdot k^{2} \cdot \frac{2}{7} \cdot h_{3}^{(1)}\left(k R_{2}\right)+\Lambda_{1} \cdot k_{l}^{2} \cdot \frac{2}{7} \cdot b_{3} \cdot j_{3}\left(k_{l} R_{2}\right) \\
& -\frac{4}{7} \cdot \mu_{1} \cdot b_{3} \cdot j_{3}^{\prime \prime}\left(k_{l} R_{2}\right)+2 \cdot \mu_{1} \cdot\left[\frac{24}{7 R_{2}} \cdot d_{3} \cdot j_{3}\left(k_{t} R_{2}\right)-\frac{24}{7 R_{2}^{2}} \cdot d_{3} \cdot j_{3}^{\prime}\left(k_{t} R_{2}\right)\right]
\end{aligned}
$$

from condition (17):

$$
-\frac{288}{7 R_{2}^{2}} \cdot d_{3} \cdot j_{3}\left(k_{t} R_{2}\right)-\frac{24}{7} \cdot d_{3} \cdot j_{3} "\left(k_{t} R_{2}\right)+\frac{48}{7 R_{2}^{2}} \cdot b_{3} \cdot j_{3}\left(k_{l} R_{2}\right)-\frac{48}{R_{2}^{2}} d_{7} \cdot j_{7}\left(k_{t} R_{2}\right)=0 ;
$$

As you can see, the system of equations became simpler then it was in case with the shell, it lost all of the terms containing the Neumann functions. Numerical values appears from integrals, which, for their turn, appears by using property of orthogonality of Legendre polynomials (as well as shifted Legendre polynomials and derivatives from them) and changes along with change of $m$.

In our case: $R_{2}=4,0 \mathrm{~m}$.

$$
\Lambda_{0}=2,25 \cdot 10^{9} \mathrm{~N} / \mathrm{m}^{2} ; \Lambda_{1}=11,2 \cdot 10^{10} \mathrm{~N} / \mathrm{m}^{2} ; \mu_{1}=8,1 \cdot 10^{10} \mathrm{~N} / \mathrm{m}^{2} ;
$$

where $\Lambda_{0}$ - modulus of volume compression; $\Lambda_{1}$ - first Lame coefficient for steel; $\mu_{1}$ - rigidity modulus.

$$
k=\frac{2 \pi v}{c} ; k_{l}=\frac{2 \pi v}{c_{l}} ; k_{t}=\frac{2 \pi v}{c_{t}} ;
$$




$$
c=1500 \mathrm{~m} / \mathrm{s} ; \quad c_{l}=5940 \mathrm{~m} / \mathrm{s} ; \quad c_{t}=3220 \mathrm{~m} / \mathrm{s} .
$$

Functional $F(\theta)$ is computing with angular step $h_{\Delta \theta}=5^{\circ}$ in the range $\theta=0^{\circ} \div 180^{\circ}$.

Same as for the shell, knowing numerical values of the angular characteristics of the scattering and the use of the Fourier transform allows us to find the form and duration of reflected impulses.

Falling impulses with $n=30$ were used during the accounts; radius of the sphere is $R_{2}=4.0 \mathrm{~m}$. On figures $12-15$ are represented pulses reflected from elastic isotropic sphere at different irradiation angles.

$\Psi_{s}(t)$

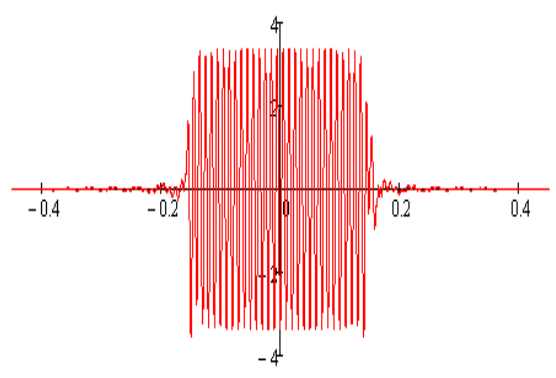

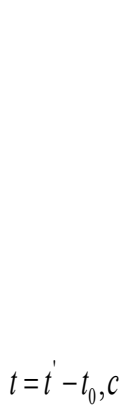
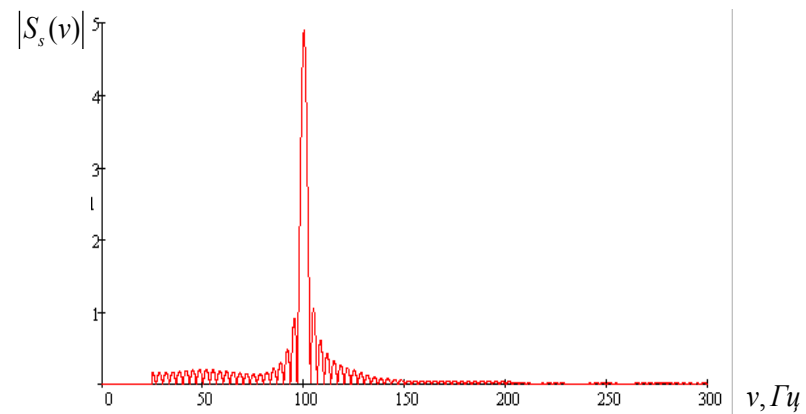

Figure 12. Impulse reflected from elastic sphere $\Psi_{s}(t)$ and its spectrum module

$$
\left|S_{s}(v)\right| \text { at angle } \theta=0^{\circ}
$$

$\Psi_{s}(t)$

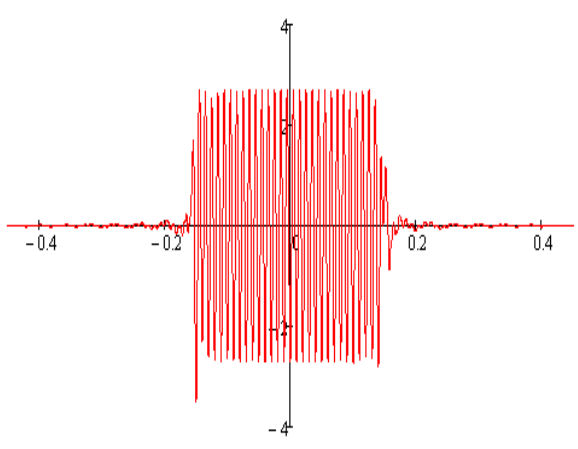

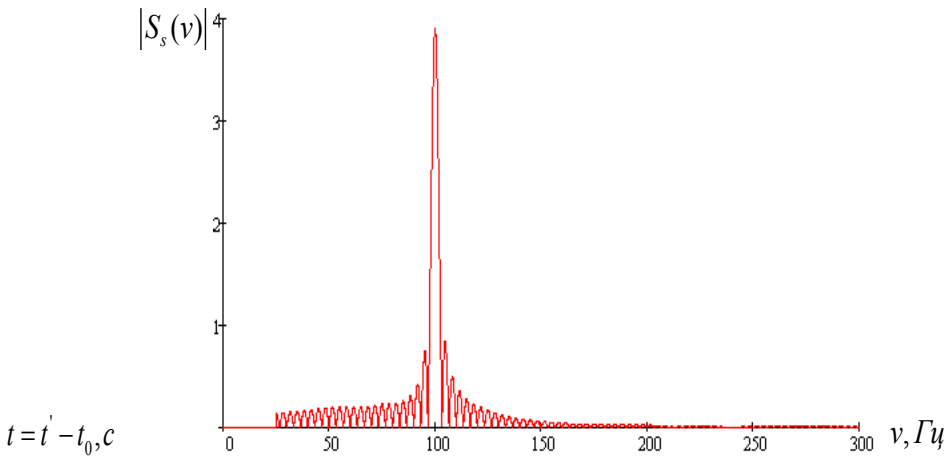

Figure 13. Impulse reflected from elastic sphere $\Psi_{s}(t)$ and its spectrum module

$$
\left|S_{s}(v)\right| \text { at angle } \theta=30^{\circ}
$$
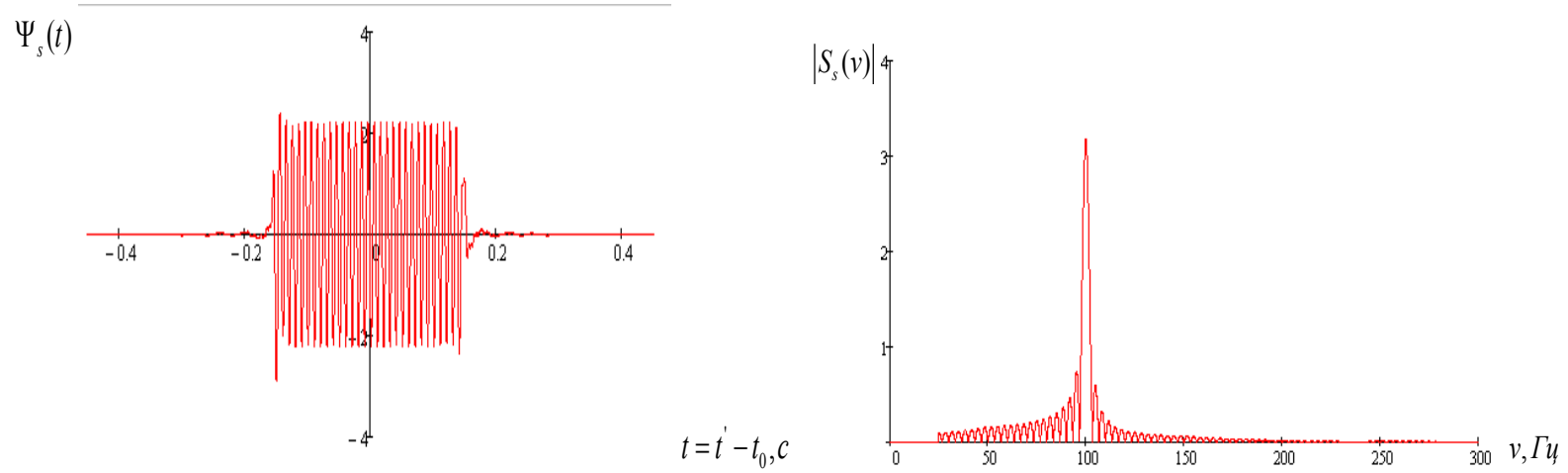

Figure 14. Impulse reflected from elastic sphere $\Psi_{s}(t)$ and its spectrum module

$$
\left|S_{s}(v)\right| \text { at angle } \theta=60^{\circ}
$$


$\Psi_{s}(t)$

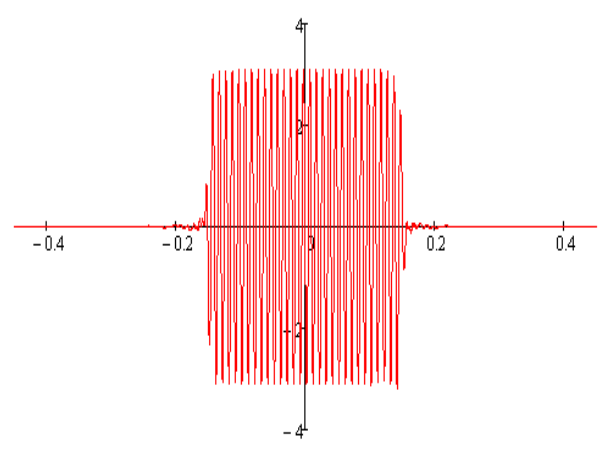

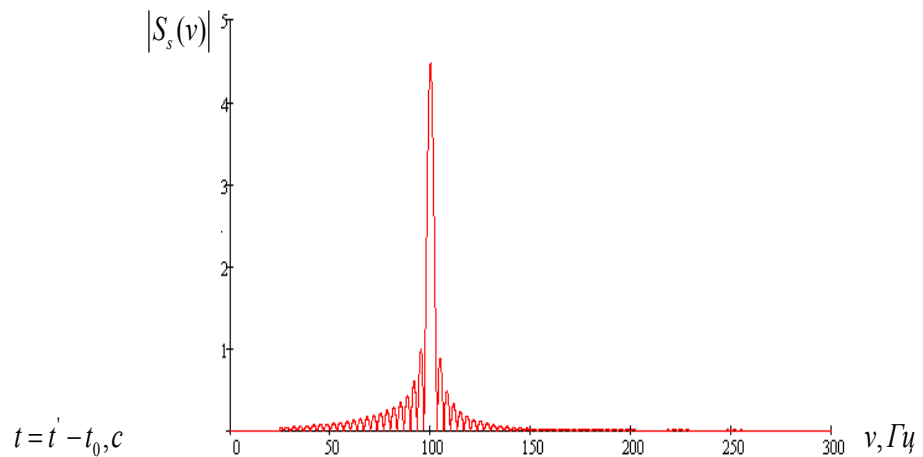

Figure 15. Impulse reflected from elastic sphere $\Psi_{s}(t)$ and its spectrum module

$$
\left|S_{s}(v)\right| \text { at angle } \theta=90^{\circ}
$$

Let's compare computed results for elastic sphere with results for sound rough ideal sphere.

$\Psi_{s}(t)$

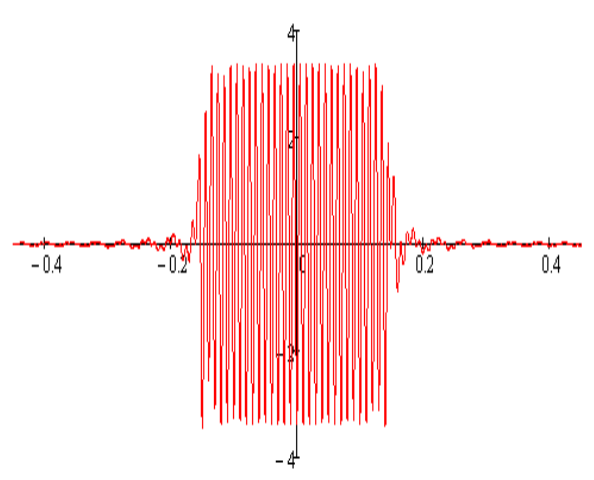

$$
t=t^{\prime}-t_{0}, c
$$

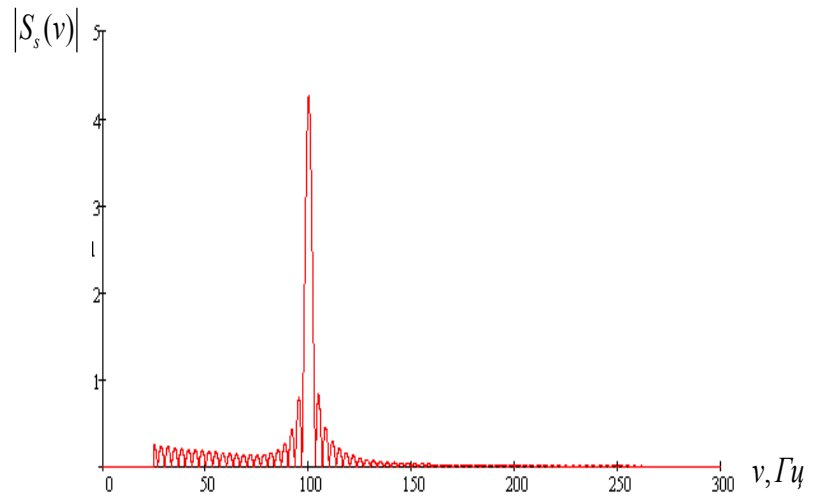

Figure 16. Impulse reflected from sound rough ideal sphere $\Psi_{s}(t)$ and its spectrum module

$$
\left|S_{s}(v)\right| \text { at angle } \theta=0^{\circ}
$$

$\Psi_{s}(t)$
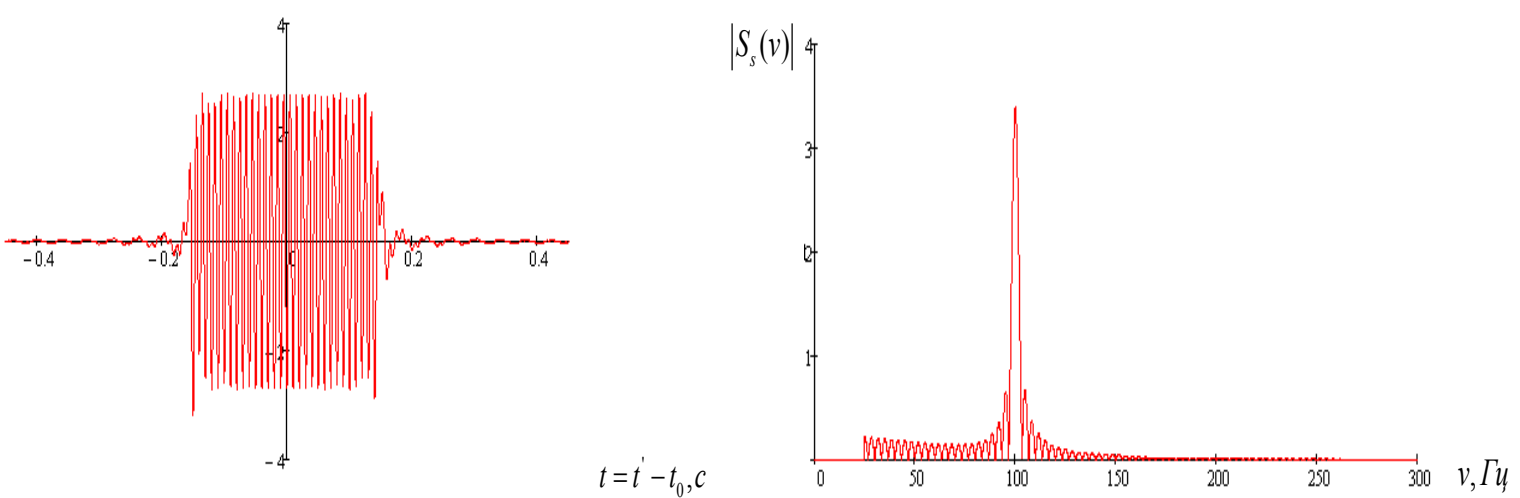

Figure 17. Impulse reflected from sound rough ideal sphere $\Psi_{s}(t)$ and its spectrum module

$$
\left|S_{s}(v)\right| \text { at angle } \theta=30^{\circ}
$$



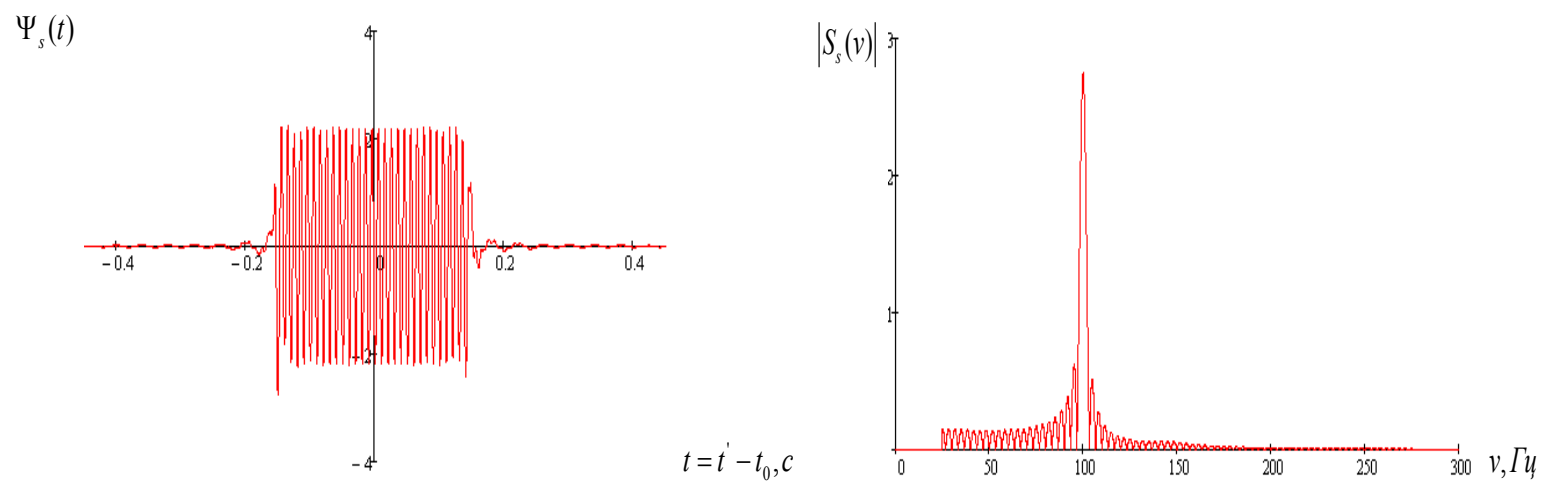

Figure 18. Impulse reflected from sound rough ideal sphere $\Psi_{s}(t)$ and its spectrum module

$$
\left|S_{s}(v)\right| \text { at angle } \theta=60^{\circ}
$$
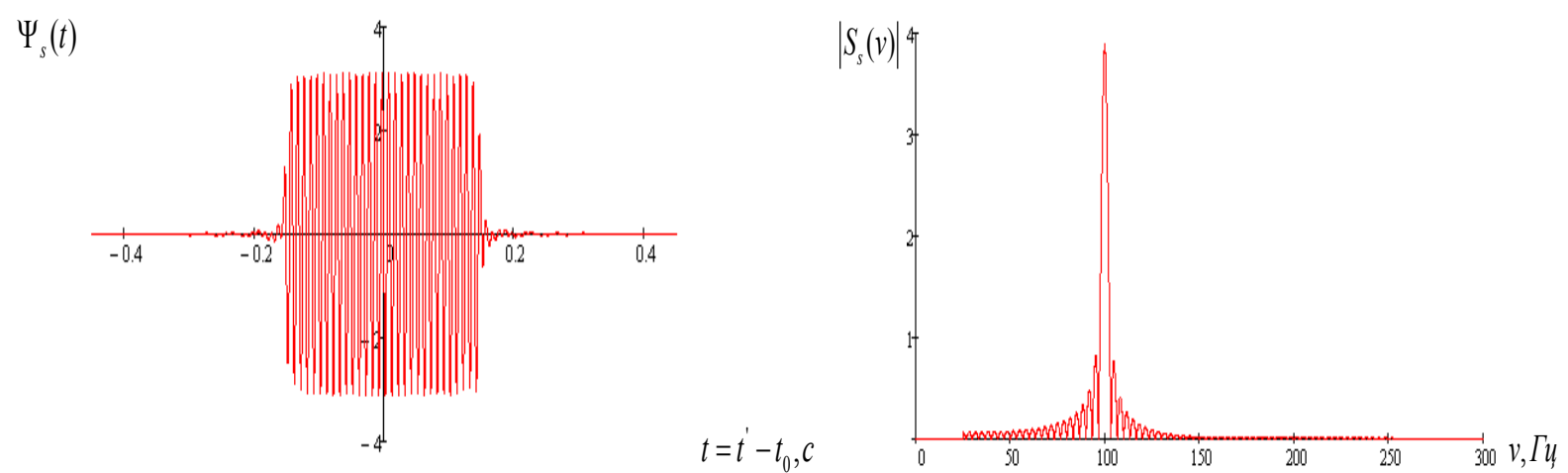

Figure 19. Impulse reflected from sound rough ideal sphere $\Psi_{s}(t)$ and its spectrum module

$$
\left|S_{s}(v)\right| \text { at angle } \theta=90^{\circ}
$$

On figures $16-19$ are represented impulses reflected from sound rough ideal sphere at different irradiation angles.

\section{Conclusions}

Using the basis of dynamic theory of elasticity were computed form and duration of impulses, which scattered on elastic spherical bodies (sphere and shell) In conclusion, it is significant that in contrast to ideal bodies, the form of impulses scattered on elastic bodies has more complicated ("broken") view. It's undoubtedly bound up with phenomena of elastic resonances which appear in elastic bodies at irradiation. Especially it can be observed by the computed results for elastic spherical shell.

\section{REFERENCES}

[1] Faran J.J. Sound scattering by solid cylinders and spheres. // J.A.S.A. 1951. V. 23. № 4. P. $405-418$.
[2] Hickling R. Analysis of echoes from solid elastic sphere in water. // J.A.S.A. 1962. V. 34. № 10. P. $1582-1592$.

[3] Hickling R. Analysis of echoes from a hollow metallic sphere in water. // J.A.S.A. 1964. V. 36. № 6. P. $1124-1137$.

[4] Sharpharets B.P. To the problem of scattering amplitude evaluation on radially-symmetric elastic inserts in perfect fluid. // Scientific industry. 2012. V. 22. № 2. P. 82-89 [in Russian].

[5] Metsaveer Y.A., Veksler N.D., Stulov A.S. Diffraction of acoustic impulses on elastic bodies, 1979, Nauka [in Russian]

[6] Shenderov E.L. Radiation and dispersion of sound, 1989, Sudostroenie [in Russian].

[7] Kleshchev. A.A., Klukin I.I. Foundations of hydroacoustics, 1987, Sudostroenie [in Russian].

[8] Kleshchev. A.A. Hydroacoustic scatterers. First publication, St. Petersburg, Shipbuilding, 1992, second publication, St. Petersburg, Prima, 2012 [in Russian]. 